\section{Fascitis eosinofílica: una causa infrecuente de aparición de edemas en Atención Primaria. A propósito de un caso}

\section{Sr. Director:}

En nuestros Centros de Salud los edemas son un motivo de consulta frecuente. En su etiología ${ }^{1}$ diferenciaremos los localizados de los generalizados. El localizado es secundario a alteraciones locorregionales; mientras que el generalizado es debido a afecciones sistémicas. Exponemos un caso clínico de interés en pacientes con etiología no aclarada.

Caso clínico: mujer de 46 años, fumadora de 30 paquetes/año, acude por dolor de 4-6 semanas de evolución, tumefacción y aparición de edemas a nivel de partes distales preferentemente extremidades inferiores. Se acompaña de astenia y artralgias a nivel grandes articulaciones, junto a síndrome del túnel carpiano. No otra sintomatología. No ingesta de fármacos o suplementos de triptófano. No exposición a tóxicos.

Exploración: temperatura $36,5^{\circ}$ C; TA $130 / 70$; pulso 89 por minuto. Edema semiduro difuso en partes distales, sobre todo de miembros inferiores que deja fóvea. No adenopatías ni lesiones cutáneas. No bocio. Resto anodino. Tinel positivo. Test de Raynaud negativo.

Analítica: hemograma: 9230 leucos (7\% eosinófilos), resto normal. VSG: 20-30. Bioquímica general, función hepática-renal, coagulación y sedimento normales. Proteínas totales: 6,8 g (29\% gammaglobulinas policlonales) TSH, $\mathrm{T} 3, \mathrm{~T} 4$, creatinquinasa y aldolasa normales. ANA, antiDNA, anti U1-nRNP, anti-Ro/SS-A, anti$\mathrm{La} / \mathrm{SS}-\mathrm{B}$, anti-Ku, anti Scl-70, anti-Sm negativos. Serología de triquina negativa.

Rx tórax y EKG: normales. Electromiograma: síndrome del túnel carpiano bilateral.
Ante la sospecha de fascitis eosinofílica se derivó al paciente a la Unidad de Diagnóstico Rápido del hospital de referencia que completó el estudio realizando una biopsia cutánea-muscular que mostró una infiltración perivascular a nivel de dermis, grasa subcutánea (llega a tabiques fibrosos).

Músculo: infiltración en epimisio y perimisio.

Tratamiento: prednisona: 60 $\mathrm{mg} /$ día con disminución progresiva de dosis y fisioterapia. A la $3^{a}$ semana lenta mejoría del edema, con remisión a los 9 meses.

Discusión: la fascitis eosinofílica es un síndrome caracterizado por tumefacción e induración de la piel y tejidos blandos subyacentes que afecta sobre todo a antebrazos y pantorrillas ${ }^{1}$. La mayoría ocurren entre la $3^{\mathrm{a}}$ y $6^{\mathrm{a}}$ década de la vida.

En muchas ocasiones la etiología es desconocida (se denomina enfermedad de Shulman) pero en un $50 \%$ casos aparece tras esfuerzo físico, o debido a tóxicos y más raramente como síndrome 
paraneoplásico $^{2,5,7}$.

Es habitual la presencia de dolor, edema, e hipersensibilidad localizados en antebrazos, piernas, manos y pies de forma simétrica. Las zonas afectas adquieren aspecto de "piel de naranja" Inicialmente la tumefacción cutánea es debida a un edema que deja fóvea, pero en fases más avanzadas se convierte en un engrosamiento elástico. Puede asociarse malestar general y fiebre. El síndrome del túnel carpiano aparece en $20-40 \%$ de casos. No es rara la presencia de morfea ${ }^{2,4}$.

Aunque poco frecuente, a veces aparece patrón restrictivo pulmonar, pleuritis, pericarditis, artritis y síndrome de Sjögren. Las contracturas articulares aparecen en 55-75\% de casos. Aparece elevación de VSG, eosinofilia periférica y aumento policlonal de $\mathrm{IgG}^{6,8}$. Ocasionalmente se ha observado ANA, factor reumatoide, anticuerpos antitiroide os y aumento de aldolasa ${ }^{6}$.

El diagnóstico de la enfermedad se basa en la clínica, eosinofilia llamativa y se confirma con la histología ${ }^{2}$. Habitualmente existe remisión completa a los 2-5 años, pero en un $10 \%$ de los casos pueden presentar entidades inmunitarias asociadas (anemia aplásica o hemolítica, trombocitopenia, síndromes mielodisplásicos y procesos linfoproliferati $\operatorname{vos})^{2,6}$.

Clínicamente se puede plante ar el diagnóstico diferencial con diferentes enfermedades que cursan con edemas generalizados, como las cardiopatías (insuficiencia cardiaca congestiva, hipertensión arterial, cor pulmonale, miocardiopatías), hepatopatía crónica difusa, nefropatías (insuficiencia renal, secundaria a diabetes mellitus y/o hipertensión arterial, glomerulonefritis, síndrome nefrótico), hipoalbuminemia, hipotiroidismo, fármacos (antagonistas del calcio, minoxidil, inhibidores del enzima conversor de la angiotensina, nitratos, alfa bloqueantes, estrógenos) y el idiopático ${ }^{3}$.

Los corticoides son los fármacos más empleados para su tratamiento. Se recomienda empezar con 40-60 mg/día de prednisona, bajando de forma progresiva la dosis cuando se produzca la mejoría ${ }^{2,5,6}$.

Sabemos de sobra que la Atención Primaria es la puerta de entrada de muchos y variados problemas de salud. En el caso planteado es fundamental su detección y la buena coordinación hospitalaria para llevar a cabo su diagnóstico, abordaje y seguimiento.

\section{J. Alins Presas, I. Caballero Humet, C. Moriana de la Olla*, J. Panisello Royo**}

Médico Especialista en Medicina Familiar y Comunitaria. ABS Santa Margarita de Montbui. UDMFC Costa de Ponent.

* Médico Residente en Medicina Familiar y Comunitaria.

** Médico Especialista en Medicina Interna. Jefe de Servicio UDR Medicina. Hospital General de Igualada. Barcelona

\section{BIBLIOGRAFÍA}

1. Fernández Barrero $C$, Antuña $C$ En: Guía de Actuación en Atención Primaria. Barcelona: Sociedad Española de Medicina Familiar y Comunitaria 1998; 460-3.

2. Saez JA. Fascitis eosinofílica y enfermedades relacionadas. An Med Interna 1999; 16: 477-83.

3. Peiro M, Calderón J. Fascitis eosinofílica. Síndrome de aceite tóxico. Síndrome eosinofilia-mialgia. En: Manual de Enf Reumáticas 1996; 369-70.

4. Jensen $E$, Hess B, Hunziker T. Eosninophilic fasciitis. Schweiz Med Wochenschr 2000; 130 : 156-60.

5. Saez JA. Eosinophilic fasciitis and related disease. An Med Interna 1999; 16: 477-83.

6. Achurra AF, Mendieta MC, Llerena JM, Benitez R. Eosinophilic Fasciitis. A report of a clinical case and the current concepts for its diagnosis and treatment. Rev Med Panama 1997; 22: 39-44.

7. Naschitz JE, Rosner I, Rozenbaum M, Zuckerman E. Rheuma- tic syndromes: clues to occult neoplasia. Semin Arthritis Rheum 1999; 29: 43-55.

8. Varga J, Kahari VM. Eosinophiliamyalgia syndrome, eosinophilic fasciitis, and related fibrosing disorders. Curr Opin Rheumatol 1997; 9: 562-70.

\section{Variabilidad clínica en la indicación de folatos} a la embarazada

\section{Sr. Director:}

La variabilidad en la indicación clínica de folatos en la gestación quedó latente en el seno de un equipo de Atención Primaria, cuyos miembros facultativos, doce médicos de Atención Primaria $y$ tres pediatras $(n=15)$, con un grado de respuesta del $100 \%$, respondieron durante una sesión de formación continuada en su centro de salud, a ítems en relación con la citada indicación. Las respuestas a las preguntas sobre si se considera eficaz la suplementación con folatos a la gestante, principio activo considerado de elección y dosis adecuada demuestran variabilidad significativa. El $20 \%$ de encuestados opina que los folatos han de prescribirse tres meses antes de la gestación, frente al $33 \%$ que piensa ha de recomendarse una vez conocida la gestación y al $40 \%$ con recomendación de iniciar la prevención desde que se planifica el embarazo. El 6,66\% no considera indicado el uso de folatos con este fin.

El $60 \%$ de los encuestados piensa que debe mantenerse la prevención farmacológica hasta el final del segundo trimestre de la gestación, frente al 26,66\% que lo recomienda hasta el final del primer trimestre de embarazo. La evidencia científica revela que la suplementación con folatos preconcepcionalmente reduce la incidencia de defectos del tubo neural (DTN) -odds ratio 0,28 , 